\title{
Thermoelectric properties of tungsten ceramics prepared from nanopowder precursors
}

\author{
DONG Liang ${ }^{1 *}$, CHEN HanJun ${ }^{1}$, GAN YingJie ${ }^{2}$, WANG Yu ${ }^{1}$, DONG Xiang ${ }^{1} \&$ PENG ShuJie ${ }^{2}$ \\ ${ }^{1}$ School of Electrical Engineering, Southwest Jiaotong University, Chengdu 610031, China; \\ ${ }^{2}$ School of Material Science and Engineering, Southwest Jiaotong University, Chengdu 610031, China
}

Received March 15, 2013; accepted May 2, 2013; published online May 28, 2013

\begin{abstract}
Tungsten trioxide ceramics were prepared from nm-sized powder precursors. Thermoelectric properties of samples were investigated. The results demonstrated that ceramics prepared in this manner have improved electrical conductivity and Seebeck coefficient values. The power factor of these samples also improved markedly, the largest power factor being $0.027 \mu \mathrm{W} \mathrm{m}^{-1} \mathrm{~K}^{-2}$ at $773 \mathrm{~K}$.
\end{abstract}

nanopowders, electrical conductivity, Seebeck coefficient, powder factor, tungsten trioxide Citation: Dong L, Chen H J, Gan Y J, et al. Thermoelectric properties of tungsten ceramics prepared from nanopowder precursors. Chin Sci Bull, 2013, 58:
2924-2926, doi: 10.1007/s11434-013-5924-x

Thermoelectric materials that convert heat into electrical energy directly have attracted much attention recently, driven by demands for clean energy sources and device coolers [1,2]. For this purpose, thermoelectric materials with high energy-conversion efficiency are widely sought. In 1997, Terasaki et al. [3] first reported the discovery of layered p-type cobalt oxides that had comparable figure of merit to conventional materials. Since then, attention has focused on metal oxides. Recently, metal oxides have been exploited as candidates for applications in thermoelectric generation because of their thermal and chemical stability in air at high temperature, oxidation resistance, reduced toxicity, easy manufacture, and low cost [4-9].

We have studied the thermoelectric properties of tungsten trioxide $\left(\mathrm{WO}_{3}\right)$ [10-12] in recent years. As is wellknown, the electrical conductivity and thermoelectric properties of $\mathrm{WO}_{3}$-based ceramics prepared by $\mu \mathrm{m}$-powders is quite low; if these ceramics are to play a role, the thermoelectric properties of $\mathrm{WO}_{3}$ require further research and improvement. With this aim, those properties of tungsten ceramics prepared from nanopowders rather than $\mu \mathrm{m}$-powders

*Corresponding author (email: ldong@ home.swjtu.edu.cn) have for the first time been investigated. We found that the thermoelectric performance of the new ceramics can be improved markedly.

$\mathrm{H}_{2} \mathrm{WO}_{4}$ and citric acid were added to liquid ammonia, and the solution was magnetically stirred for $10 \mathrm{~h}$ to produce a yellow colored colloidal solution. This solution was placed in an infrared baking oven and baked until it became a black gelatum. The gel was sintered at $823 \mathrm{~K}$ for $2 \mathrm{~h}$ at a rate of heating of $3{ }^{\circ} \mathrm{C} \mathrm{min}{ }^{-1}$ and cooled in the furnace to produce the nanometer tungsten trioxide powders. After milling in a mortar of agate for $5 \mathrm{~h}$, the mixed powder was pressed into wafers $10 \mathrm{~mm}$ in diameter and $1.2 \mathrm{~mm}$ thick under a pressure of about $300 \mathrm{MPa}$. The pellets were sintered in a muffle furnace at $1000^{\circ} \mathrm{C}$ for $1 \mathrm{~h}$ in air.

The surface microstructure of the composite was examined using a scanning electron microscope (SEM, FEI QUANTA200, Netherlands). For the SEM analysis and Energy Dispersive X-Ray Spectroscopy (EDX), the samples were cleaned with acetone, mounted, and gold-coated to prevent charging. The crystalline phases of the sintered sample were identified using a X-ray diffractometer (XRD, 7602 EA Almelo, Netherlands) under the following experimental conditions: $\mathrm{Cu} \mathrm{K} \alpha, \lambda=0.15406 \mathrm{~nm}, 40 \mathrm{kV}, 40 \mathrm{~mA}$, 
$20^{\circ} \leqslant 2 \theta \leqslant 60^{\circ}$. The electrical conductivity and thermoelectric power over the temperature range from 300 to $773 \mathrm{~K}$ were calculated from thermoelectric voltage data (Series 2400, Keithley Instruments, Cleveland, $\mathrm{OH}$ ) and the temperature difference between the two ends of the sample.

A SEM micrograph (Figure 1) of a $\mathrm{WO}_{3}$ ceramic prepared from nanopowders shows that the grain size of the sample is about $1 \mu \mathrm{m}$, smaller than the samples prepared with $\mu \mathrm{m}$-powders. The phase structure of the samples is shown in Figure 2. The JCPDS database of crystallographic entries suggest that the triclinic $\left(\delta-\mathrm{WO}_{3}\right)$ and monoclinic $\left(\gamma-\mathrm{WO}_{3}\right)$ phases of $\mathrm{WO}_{3}$ are coexisting. The result is similar to the undoped samples prepared from $\mu \mathrm{m}$-powders [13].

The temperature dependence of the electrical conductivity $(\sigma)$ for the sample (see log-normal plot in Figure 3 ) indicate that the curves of $\ln \sigma$ against $1000 / T$ exhibit near linear characteristics for $T>300^{\circ} \mathrm{C}$, suggesting approximately semiconductive behavior. However, the variation in the electrical properties for $T<300^{\circ} \mathrm{C}$ does not obey Arrhenius' law:

$$
\sigma=\sigma_{0} \exp \left(-\frac{E_{\alpha}}{k T}\right)
$$

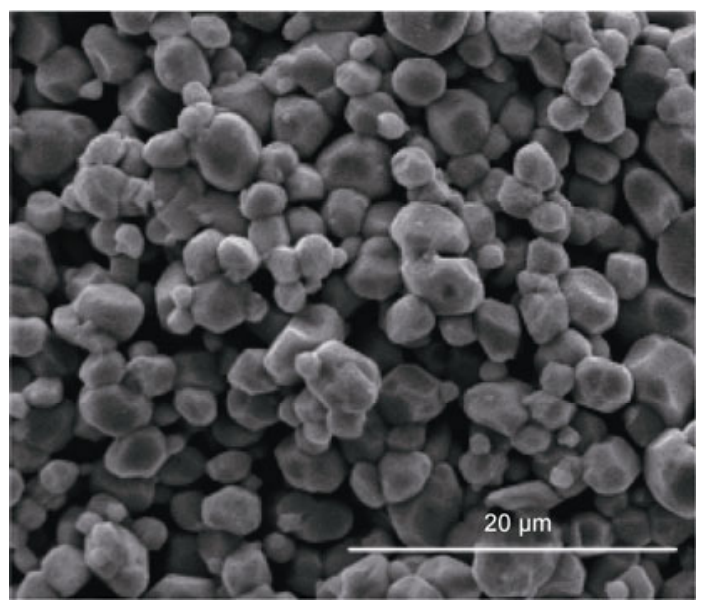

Figure 1 SEM micrograph of tungsten trioxide ceramic prepared from nanopowder.

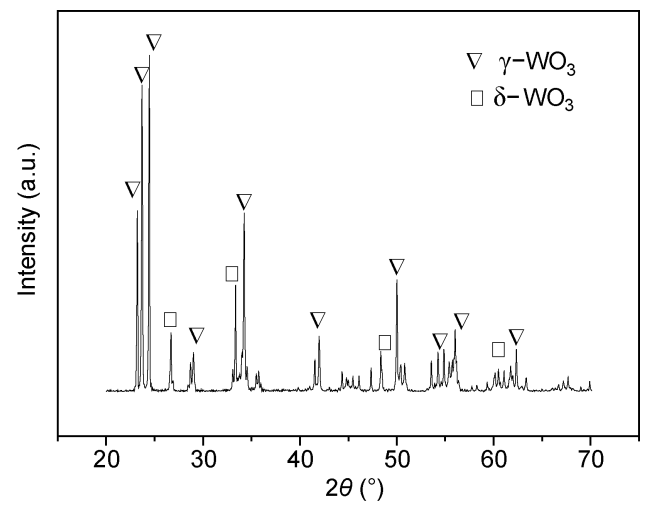

Figure 2 XRD patterns of the sample.

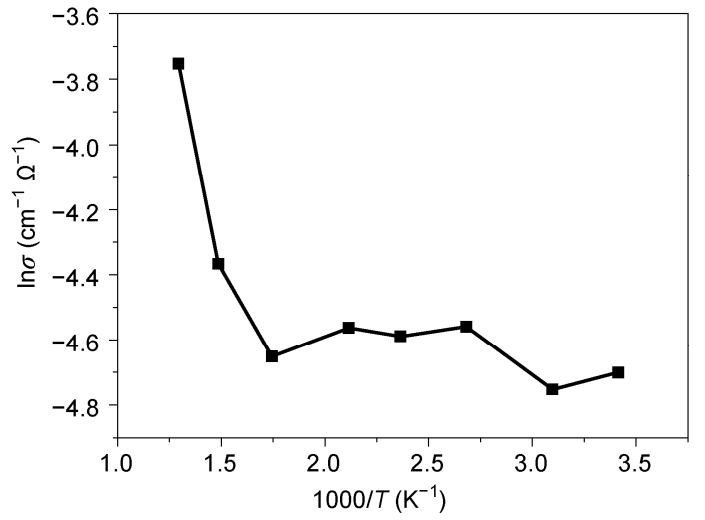

Figure 3 Temperature dependence of the electrical conductivity of the sample.

where $\sigma$ is the electrical conductivity, $\sigma_{0}$ is a constant, $k$ is the Boltzmann constant, $T$ is the absolute temperature, and $E_{\alpha}$ is activation energy. Clearly, an important change in the electrical characteristics is occurring in the ceramics as temperature increases above room temperature; other physical processes are influencing electrical transport besides heat activation. The variation of the activation energy is due to the $\mathrm{WO}_{3}$ phase transition at temperatures of about $300^{\circ} \mathrm{C}$ where $1000 / T$ is $1.7 \mathrm{~K}^{-1}$. The result also demonstrates that the electrical conductivity of the sample prepared from nanopowders is two orders of magnitude greater than the sample prepared from $\mu \mathrm{m}$-powders [10].

Figure 4 presents the temperature dependence of the Seebeck coefficient $(S)$ for the sample. The absolute value of $S$ increases as temperature increases, but its values are all negative over this temperature range, indicating n-type conduction. In general, the Seebeck coefficient decreases with increasing electrical conductivity $[8,14]$. According to a simplified broadband model, the Seebeck coefficient for extrinsic n-type semiconductor devices with negligible hole conduction can be expressed as [15]: $S=-(k / e)\left[\ln \left(N_{v} / n\right)+A\right]$, where $k$ is the Boltzmann constant, $n$ is the electron concentration, $e$ is the electric charge of the carrier, $N_{v}$ is the density of state, and $A$ is a transport constant, typically $0 \leqslant A \leqslant 2$.

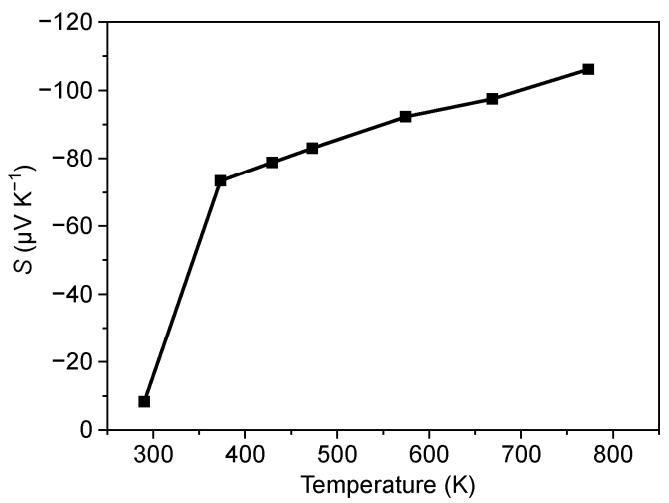

Figure 4 Temperature dependence of the Seebeck coefficients of the sample. 


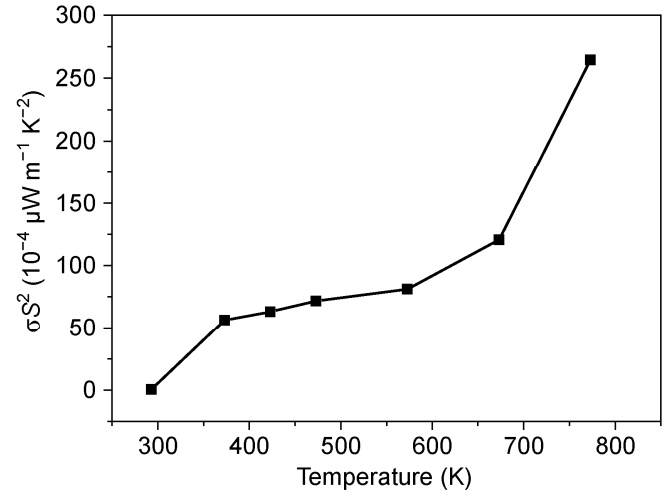

Figure 5 Temperature dependence of the power factor of the sample.

In general, with increasing electrical conductivity, the Seebeck coefficient decreases $[8,16]$. Moreover, grain size also affects the value of the Seebeck coefficient. Gao et al. [17] reported that the Seebeck coefficient increased as size decreased. The largest value for the Seebeck coefficient is $-106.2 \mu \mathrm{V} \mathrm{K}^{-1}$, which is larger than the samples made by $\mu \mathrm{m}$-powder [11].

From Figures 3 and 4, we see the electrical conductivity and the Seebeck coefficient of the sample prepared from nanopowders were larger than that prepared from $\mu \mathrm{m}$ powders [10-12]. A plot of the thermoelectric power factor $\left(\sigma S^{2}\right)$ of the sample, as a function of temperature (Figure 5) shows enhancement as temperature rises. At $773 \mathrm{~K}$, the sample attains its largest power factor, $0.027 \mu \mathrm{W} \mathrm{m} \mathrm{K}^{-1}$.

The thermoelectric properties of $\mathrm{WO}_{3}$ ceramics prepared from nanopowders have been investigated. The results demonstrate that the electrical conductivity and the Seebeck coefficient are higher than for undoped ceramics prepared from $\mu \mathrm{m}$-powders. The sample obtains its largest power factor of $0.027 \mu \mathrm{W} \mathrm{m}{ }^{-1} \mathrm{~K}^{-2}$ at $773 \mathrm{~K}$. Thus, nanopowder preparations can provide improved thermoelectric properties of $\mathrm{WO}_{3}$ ceramics that might offer superior candidates for applications in thermoelectric generation.

This work was supported by the National Natural Science Foundation of China (50772092) and the Fundamental Research Funds for the Central Universities (2682013CX014).

Mahan G D. Solid State Phys, 1998, 51: 81-157

Vining C B, Bell L E. Science, 2008, 321: 1457-1461

3 Terasaki I, Sasago Y, Uchinokura K. Phys Rev B, 1997, 56: 12685-12687

4 Hossain M S, Islam R, Shahjahan M, et al. J Mater Sci Mater Electron, 2008, 19: 1114-1121

5 Ohta S, Nomura T, Ohta H, et al. J Appl Phys, 2005, 97: 034106

6 Androulakis J, Migiakis P, Giapintzakis J. Appl Phys Lett, 2004, 84: 1099-1101

7 Berardan D, Byl C, Dragoe N. J Am Ceram Soc, 2010, 93: 2352-2358

8 Li F, Li J F. Ceram Int, 2011, 37: 105-110

9 Park K, Jang K U, Kwon H C, et al. J Alloys Compd, 2006, 419: 213-219

10 Wang H Q, Hua Z Q, Peng S J, et al. Physica B, 2011, 406: 4183-4187

11 Wang $\mathrm{H}$ Q, Hua Z Q, Peng S J, et al. Ceram Int, 2012, 38: 1133-1137

12 Dong X, Wang H Q, Hua Z Q, et al. J Mater Sci, 2012, 23: 1210-1214

13 Wang Y, Yao K L, Liu Z L. J Mater Sci Lett, 2001, 20: 1741-1743

14 Park K, Seong J K, Nahm S. J Alloys Compd, 2008, 455: 331-335

15 Noh W, Shin Y, Kim J, et al. Solid State Ionics, 2002, 152-153: 827-832

16 Bhandari C M, Rowe D M. Optimization of Carrier Concentration. Boca Raton: CRC Press, 1995. 43-46

17 Gao Y W, He Y Z, Zhu L L. Chin Sci Bull, 2010, 55: 16-21

Open Access This article is distributed under the terms of the Creative Commons Attribution License which permits any use, distribution, and reproduction in any medium, provided the original author(s) and source are credited. 